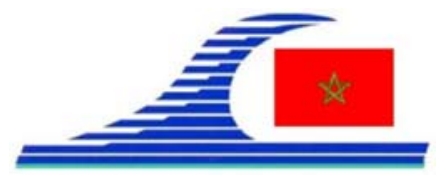

Conférence Méditerranéenne Côtière et Maritime EDITION 2, TANGER, MAROC (2011)

Coastal and Maritime Mediterranean Conference

Disponible en ligne - http://www.paralia.fr - Available online

\title{
Un programme opérationnel d'identification des modes de gestion durable des sédiments marins : le projet SEDI.MAR.D. 83
}

\author{
Jean-Luc AQUA ${ }^{1}$, Pierre BOISSERY ${ }^{2}$, Claude ALZIEU ${ }^{3}$
}

1 Chef du projet SEDI.MAR.D. 83, Conseil Général du Var, 390 Av des Lices, 83076 Toulon Cedex, France. jaqua@cg83.fr

2. Agence de l’Eau Rhône Méditerranée Corse, Délégation de Marseille, Immeuble le Noailles / 62, La Canebière, 13001 Marseille, France.

Pierre.BOISSERY@eaurmc.fr

3. Chef du Groupe d'Experts Scientifiques du projet SEDI.MAR.D 83, 190 rue Pastenagues, 34470 Perols, France.

claude.alzieu@orange.fr

\section{Introduction}

L'accumulation de sédiments dans les ports, les chenaux d'accès, les canaux, les rivières et les fleuves empêchent la circulation des bateaux et perturbent les équilibres physico-chimiques des masses d'eaux. Pour permettre le fonctionnement et le développement des établissements portuaires mais également participer à l'entretien et la restauration des milieux naturels aquatiques, les gestionnaires de ces derniers doivent entreprendre des opérations de dragage.

Les pratiques systématiques de rejet en mer présentent un risque fort de dispersion de produits écotoxiques pour le milieu marin. Elles sont, de fait, soumises à des législations nationales, européennes et internationales de plus en plus contraignantes.

Du fait du niveau de contamination de leurs sédiments, de nombreux ports de la côte méditerranéenne ont à trouver une alternative aux immersions. Ils sont donc confrontés au double problème de l'absence de solutions techniques suffisantes et de l'absence d'un contexte réglementaire précis sur le devenir à terre des sédiments.

Toutefois l'ampleur et la complexité des solutions à identifier, le souci d'apporter des réponses concertées, cohérentes dans un cadre d'acceptabilité national et communautaire, et la volonté de capitaliser sur ces problématiques ont conduit à rechercher les meilleures coopérations possibles entre les gestionnaires portuaires et institutionnels.

L’implication des principaux experts du monde de la recherche s'est également avérée indispensable pour répondre aux questionnements scientifiques complexes : sécurisation technique, environnementale et sanitaire, paramètres permettant de maîtriser les différents modes de gestion susceptibles d’être envisagés. 
La connaissance de la Mer :

un vecteur du développement durable en Méditerranée

A travers la mise en place d'un Contrat d'Objectif départemental, le Conseil Général du Var a pris l'initiative d'un projet coopératif, pluridisciplinaire et coordonné, permettant de produire les premières données nécessaires à la définition de scénarios conformes aux grands principes du Développement Durable applicables à la gestion à terre des sédiments: le programme SEDI.MAR.D. 83.

\section{Le programme SEDI.MAR.D. 83}

\subsection{Des objectifs ambitieux}

Afin d'apporter les premiers éléments de réponses opérationnelles à une problématique généralisée, soulignée lors des Grenelles de l'Environnement et de la Mer, SEDI.MAR.D 83 a regroupé, dans une approche de projet partenarial, dix maître d'Ouvrages gestionnaires portuaires français et italiens confrontés à la problématique de gestion à terre des sédiments. Dès son origine, le programme a pris l'attache du Ministère de l'Environnement (MEDDTL).

Les objectifs principaux de l'opération SEDI.MAR.D 83 ont consisté à mettre en place les bases solides de connaissances susceptibles de permettre, à court terme, des propositions d'outils aux gestionnaires des zones maritimes, dont un guide méthodologique et une base de données de référence.

\subsection{Une approche opérationnelle mutualisée de gestionnaires portuaires}

Pour pouvoir définir les différents modes de gestion envisageables il apparaît indispensable de définir et d'associer des voies de valorisation et des solutions de stockage en fonction de la nature qualitative et quantitative des différentes fractions constitutives du sédiment, objectif technique fort de SEDI.MAR.D 83.

Ainsi, pour apporter les premières réponses à cette problématique technique de filières multiples, il a été nécessaire d'impulser une dynamique de projet autour d'un véritable programme opérationnel basé sur des situations réelles de sites portuaires en difficulté et en ciblant les données à produire avec la mise en place d'un démonstrateur de traitements.

Le programme SEDI.MAR.D 83, dont la maîtrise d'Ouvrage et la maîtrise d'œuvre ont été assurées par le Département du Var, a mutualisé les actions des gestionnaires suivants : la Marine Nationale, La Chambre de Commerce et d'Industries du Var, le Conseil Général des Alpes Maritimes, La Communauté Urbaine Marseille Provence Métropole, Le Conseil Général du Finistère, La région italienne Emilia Romagna ainsi que les Commune du Lavandou, de Sanary-sur-Mer et de Bandol.

Il a été construit autour de 14 missions coordonnées pour un montant total d'environ 2 M€ co-financé à 70\% avec notamment le soutien de l'Agence de l'Eau Rhône Méditerranée Corse, l’Ademe et la Région Provence Alpes Côte d’Azur. 
L’intérêt de l'approche partenariale entre différents gestionnaires portuaires confrontés à une même problématique repose d'une part sur une mutualisation des moyens mais également sur l'éventail qualitatif des sédiments qui ont pu être caractérisés et traités. En effet, un des paramètres majeur du "déchet-sédiment" est bien la variabilité du gisement.

La mission principale a consisté à réaliser un site industriel de traitement à une échelle pilote de grande taille sur un site portuaire de 1 hectare dans la rade de Toulon. Le prestataire industriel spécialisé dans le traitement des sédiments, la Société ExtractEcoterres a mis en œuvre, pendant 18 mois, l'ensemble des combinaisons de traitement sur des volumes unitaires de $200 \mathrm{~m}^{3}$ dragués sur les différents sites portuaires des partenaires du projet.

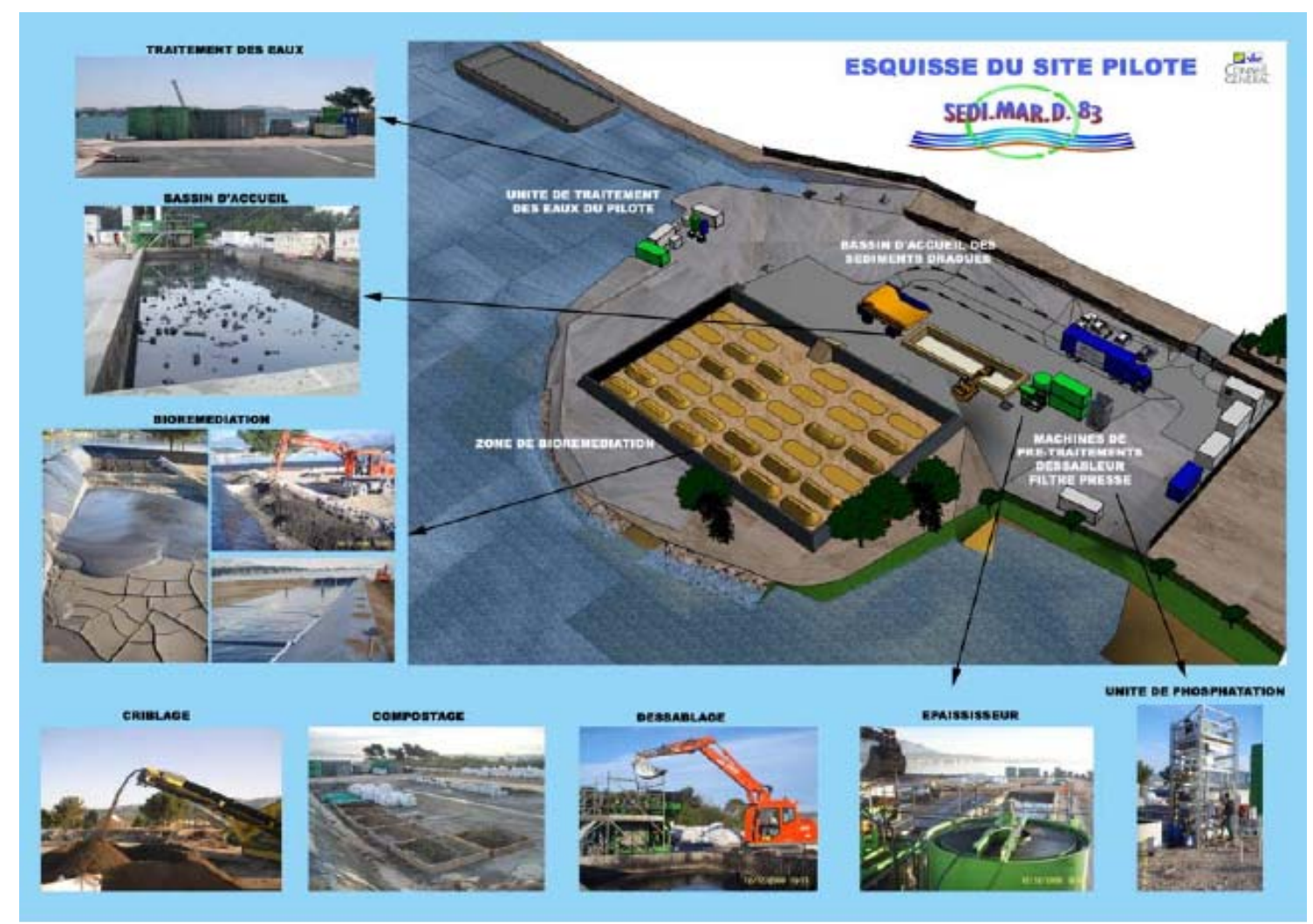

Figure 1. Esquisse 3D de la plate-forme pilote SEDI.MAR.D. 83

et fonctionnement du site.

\subsection{Un projet partenarial entre gestionnaires opérationnels et acteurs scientifiques}

Les gestionnaires portuaires ne peuvent pas apporter, seuls, toutes les réponses techniques, environnementales et sanitaires indispensables à l'évaluation des impacts des opérations de valorisations. L'approche scientifique est indispensable à l'amélioration des connaissances, à long terme, sur la problématique. 
La connaissance de la Mer :

un vecteur du développement durable en Méditerranée

De par le caractère innovant du projet et la nécessité de répondre à des problématiques nouvelles, il a été décidé de mettre en place un Groupe d’Experts Scientifiques (GES) indépendant pour accompagner le déroulement des différentes missions.

Le GES a été constitué par une équipe de scientifiques spécialisés sur les différentes disciplines du domaine. Ainsi, ont été mobilisés des experts de l'Ifremer à Sète, l'UT2A à Pau, l'Université de Caen, le CEMAGREF à Lyon, le BRGM à Nantes ainsi que de l’Ecole des Mines à Douai.

Un programme structuré en quatre axes de travail :

- Axe 1 : Caractérisations des sédiments

Caractérisations menées avec une approche adaptée à la gestion terrestre et dans le cadre de la définition des potentialités de valorisation. En particulier, définition d'un protocole d'évaluation de la dangerosité des sédiments non immergeables.

- Axe 2 : Opérations de traitements de 10 sites distincts (axe majeur de SEDI.MAR.D 83)

Opération de travaux menée à l'échelle du démonstrateur pilote, sur une plate-forme en rade de Toulon où a été testée la robustesse de plus de 60 combinaisons de traitements.

- Axe 3 : Analyses des possibilités de filières de gestion

Un outil d'analyse multicritères a été développé afin de permettre aux décideurs d'analyser les avantages et inconvénients des différentes possibilités de gestion.

- Axe 4 : Etudes d'approfondissement

En cours d'opération des questions scientifiques complexes sur le comportement lors des traitements des sédiments sont apparues. Elles ont fait l'objet d'études spécifiques.

\subsection{Des résultats essentiels}

Les données produites lors des phases de caractérisation et à toutes les étapes des différentes combinaisons de traitement ont été regroupées à l'aide de deux outils, une banque de données et de méta-données numériques comportant plus de 2700 fiches analytiques soit environ 37000 données produites et une banque d'échantillons prélevés lors des traitements dénommée "sédimentothèque" avec plus de 520 produits stockés.

Au delà des résultats techniques obtenus, ce projet a aussi mis en évidence l'intérêt d'un travail partenarial entre gestionnaires confrontés à une même problématique et acteurs de la recherche qui a abouti à la réalisation d'un outil de gestion global : l'analyse multicritère des scénarios. A noter également le soutien des populations et des associations de défense de l'environnement à une approche ressentie comme une contribution forte à la protection du milieu aquatique et du littoral. 


\section{Conclusion et perspectives}

Les résultats du programme SEDIMARD 83, dont une partie est publiée dans la revue et sur le site Paralia (SANNIER et al., 2008 ; AQUA et al., 2009 ; MAMINDY-PAJANY et al., 2009 ; MANCIOPPI et al., 2009 ; SANNIER et al., 2009 ; SEBY et al., 2009, LEVACHER \& DHERVILLY, 2010 ; LEVACHER et al., 2011) sont dans bien des cas originaux et encourageants quand aux possibilités de traitement à terre des sédiments contaminés. Un projet de diffusion élargie des résultats sera mené en 2012 par l'Insa de Lyon et Insavalor.

L'opération a permis d'obtenir des informations techniques et environnementales indispensables à la résolution de la problématique de gestion et de proposer des protocoles propres à compléter la réglementation. Le travail partenarial entre les gestionnaires portuaires et les acteurs du monde de la recherche s'est avéré être un facteur déterminant. Enfin, les solutions pour une gestion durable des sédiments contaminés, conformes aux attentes sociétales et aux obligations réglementaires, sont maintenant appréhendées par tous les maîtres d'ouvrage. Elles sont basées sur une approche multi-filières et s'adaptent aux caractéristiques qualitatives et quantitatives des potentialités territoriales.

La sécurisation technique et environnementale des filières de gestion, simplement abordée lors du programme SEDI.MAR.D 83, apparaît donc comme une priorité.

L'approche multi-partenaires développée par SEDI.MAR.D 83 qui a permis d'atteindre des objectifs jugés très ambitieux au départ pourrait être mise à profit pour initier et conduire des programmes futurs tels que Sedimatériaux ou Sediplateforme.

Au-delà des aspects techniques et réglementaires, la mise en place d'un dispositif économique permettant d'accompagner financièrement les ports dans la mise en œuvre des solutions de traitement identifiées lors du programme SEDI.MAR.D 83 demeure l'un des enjeux les plus importants auquel est confronté aujourd'hui la démarche. Le principe pollueur-payeur est malheureusement difficile à appliquer dans la majorité des cas, la pollution à gérer actuellement résultant d'un historique de comportements de plusieurs décennies.

\section{Références bibliographiques}

AQUA J.-L., BOISSERY P., ALZIEU C. (2009). Un programme opérationnel d'identification des modes de gestion durable des sédiments marins : le projet SEDI.MAR.D. 83. Revue Paralia, $\mathrm{n}^{\circ}$ 2, pp s1.1-s1.12. doi:10.5150/revue-paralia.2009.s01

LEVACHER D., DHERVILLY P. (2010). Déshydratation mécanisée in situ de sédiments fraîchement dragués ou mis en dépôts : le projet SEDIGATE® I. Journées Nationales Génie Côtier - Génie Civil, Les Sables d'Olonne, pp 859-868. doi:10.5150/jngcgc.2010.093-L 
La connaissance de la Mer :

un vecteur du développement durable en Méditerranée

LEVACHER D., SANCHEZ M., DUAN Z., LIANG Y. (2011). Valorisation en unité pilote de sédiments méditerranéens : étude des caractéristiques géotechniques et de la perméabilité. Revue Paralia, Vol. 4, pp 4.1-4.20. doi:10.5150/revue-paralia.2011.004

MAMINDY-PAJANY Y., HUREL C., MARMIER N., ROMÉO M. (2009). Traitement d'un sédiment portuaire contaminé par bio-remédiation et stabilisation géochimique. Conférence Méditerranéenne Côtière et Maritime, Hammamet, Tunisie, pp 127-132. doi:10.5150/cmcm.2009.032-9

MANCIOPPI L., BENARD A., HENNEBERT P., HAZEBROUCK B. (2009). Sédiments de dragage : variabilité des sédiments, retour d'expérience sur les pratiques de dragage et sur la valorisation à terre en France. Conférence Méditerranéenne Côtière et Maritime, Hammamet, Tunisie, pp 133-134. doi:10.5150/cmcm.2009.033-6

SANNIER L., LEVACHER D., JOURDAN M. (2008). Discrimination économique de méthodes de traitements de sédiments marins contaminés à l'aide de liants hydrauliques. Journées Nationales Génie Côtier - Génie Civil, Sophia Antipolis, pp 821-830. doi:10.5150/jngcgc.2008.077-S

SANNIER L., LEVACHER D., JOURDAN M. (2009). Approche économique et validation de méthodes de traitements aux liants hydrauliques de sédiments marins contaminés. Revue Paralia, $\mathrm{n}^{\circ}$ 2, pp s2.1-s2.15. doi:10.5150/revue-paralia.2009.s02

SEBY F., BENOIT-BONNEMASON C., TESSIER E., ALZIEU C., AQUA J.-L., SANNIER L., DONARD O.F.X. (2009). Etude de l'évolution des formes chimiques des métaux dans des sédiments marins dragués stockés à terre. Revue Paralia, $\mathrm{n}^{\circ} 2$, pp s3.1-s3.12. doi:10.5150/revue-paralia.2009.s03 